\title{
Spectral decomposition of optimal asset-liability management
}

\author{
Marc Decamps ${ }^{a}$ Ann De Schepper ${ }^{\text {b,* }}$, Marc Goovaerts ${ }^{\text {a,c }}$ \\ ${ }^{a}$ K.U.Leuven, FETEW, Naamsestraat 69, B-3000 Leuven, Belgium \\ ${ }^{\mathrm{b}}$ University of Antwerp, Faculty of Applied Economics, Prinsstraat 13, 2000 Antwerp, Belgium \\ ${ }^{\mathrm{c}}$ University of Amsterdam, Roetersstraat 11, NL-1018 WB Amsterdam, the Netherlands
}

\begin{abstract}
This paper concerns optimal asset-liability management when the assets and the liabilities are modeled by means of correlated geometric Brownian motions as suggested in Gerber and Shiu (2003). In a first part, we apply singular stochastic control techniques to derive a free boundary equation for the optimal value creation as a growth of liabilities or as dividend payment to shareholders. We provide analytical solutions to the HJB optimality equation in a rather general context. In a second part, we study the convergence of the cash flows to the optimal value creation using spectral methods. For particular cases, we also provide a series expansion for the probabilities of bankruptcy in finite time.
\end{abstract}

Key words: asset-liability management, HJB principle, local time, spectral theory, free boundary problem

\section{Introduction}

In a recent paper, Gerber and Shiu (2003) propose to model the asset $X_{1}(t)$ and liability $X_{2}(t)$ values of a firm by means of correlated geometric Brownian motions. Using martingale arguments, they are able to derive an analytical expression for the dividend pay-out needed to maintain the firm value $X(t)=\left(X_{1}(t), X_{2}(t)\right)$ in the cone

$$
O:=\left\{\bar{x}=\left(x_{1}, x_{2}\right) \in \mathbb{R}^{2}: x_{1} \leq \lambda x_{2} \text { and } x_{1}>\lambda_{1} x_{2}\right\}
$$

\footnotetext{
* Corresponding author.

Email addresses: marc.decamps@fortis.com (Marc Decamps), ann.deschepper@ua.ac.be (Ann De Schepper), marc.goovaerts@econ.kuleuven.be (Marc Goovaerts).
}

Preprint submitted to Journal of Economic Dynamics and Control 15 September 2008 
with $\lambda>\lambda_{1}$, imposing bankruptcy when the firm value hits the barrier

$$
e\left(\lambda_{1}\right):=\left\{\bar{x}=\left(x_{1}, x_{2}\right) \in \mathbb{R}^{2}: x_{1}=\lambda_{1} x_{2}\right\}
$$

for the first time. Maximizing the discounted dividend accumulated until ruin time $\tau=$ $\inf \left\{t \geq 0: X(t) \in e\left(\lambda_{1}\right)\right\}$, Gerber and Shiu (2003) provide an optimal value of $\lambda$ but conjecture the optimality over all possible dividend strategies. In this paper, we prove that the results of Gerber and Shiu (2003) are optimal only if the asset value of the firm is controlled, and we generalize the results to strategies allowing to control the liability values together with the asset values.

Asset-Liability Management (ALM) techniques concern the dynamic control of the balance sheet. It consists of controlling the positive and negative cash flows generated by the company activity in order to narrow the difference between assets and liabilities while maximizing the creation of value. The dynamic control of the firm value as exposed in this paper is to some extent not coherent with common standard financial theories. In asset pricing theory as pioneered by Miller and Modigliany (1958), the firm value is not affected by the dividend pay-out or by the methods of financing activities. Contrary to this, the model exposed in the present paper allows to invest the profits or to pay dividends to shareholders by modifying the dynamic of the firm asset value. Although the model is not consistent with the results of Miller and Modigliany (1958), it provides a tractable framework to study and compare the risks involved by a set of optimal assetliability management policies. The problem is classical in actuarial mathematics and finds natural applications to manage the solvency of insurance companies, isolated business lines or pension funds. A similar framework is proposed in Hojgaard and Taksar (2002). As far as we know, the literature on ALM only considers the surplus process of the assets over the liabilities. Without claiming any exhaustiveness, we refer to Asmussen and Taksar (1997), Hojgaard and Taksar (1999), Hojgaard (2002), Hubalek and Schachermayer (2004), Gerber and Shiu (2003, 2004), Rudolf and Ziemba (2004), Grandits et al. (2007), or Hoevenaers et al. (2008). It implicitly restricts the ALM committee to act on the asset value of the firm by the payment of dividend to shareholders, and to optimize these dividends. However, it is more realistic to allow the ALM committee to reinvest into new lines of business and raise liabilities when the assets grow faster than the liabilities.

In this paper, the controlled firm value satisfies

$$
d X_{i}(t)=\mu_{i} X_{i}(t) d t+\sigma_{i} X_{i}(t) d B_{i}(t)-\eta_{i} d C(t) ; \quad X(0)=\bar{x} ; \quad i=1,2
$$

where $\{B(t), t \geq 0\}$ is a bivariate Brownian motion with correlation $0 \leq \rho \leq 1$ and $\bar{\eta}=\left(\eta_{1}, \eta_{2}\right)$ is a constant unit vector. The process $X_{1}$ corresponds to the assets, and $X_{2}$ to the liabilities. Throughout the paper, $\left(\sigma_{i, j}^{2}\right)$ stands for the covariance matrix

$$
\left(\sigma_{i, j}^{2}\right)=\left(\begin{array}{cc}
\sigma_{1}^{2} & \rho \sigma_{1} \sigma_{2} \\
\rho \sigma_{1} \sigma_{2} & \sigma_{2}^{2}
\end{array}\right) .
$$

In general, a control process is a stochastic process $\{C(t), t \geq 0\}$, which is progressively measurable. In this paper, we use a more strict definition, and we also assume that the 
control process $\{C(t), t \geq 0\}$ is a non-negative non-decreasing adapted process started at 0 right continuous with left limit and admits the following cumulative representation

$$
C(t)=\int_{0}^{t} a(s) d s
$$

where the process $\{a(t), t \geq 0\}$ can be unbounded. Remark that the assumptions on $C($. do not imply the continuity of the control with respect to $t$, and thus $C($.$) can have$ discontinuities $\Delta C(t)=C(t)-C\left(t_{-}\right)$.

The ALM committee modifies both the assets and the liabilities in a given ratio $\eta_{1} / \eta_{2}$ (a change of $\Delta X_{1}(t)$ in the assets implies a change $\Delta X_{2}(t)=\frac{\eta_{1}}{\eta_{2}} \Delta X_{1}(t)$ in the liabilities). It is clear that $\eta_{1}$ is positive (e.g. through the payment of dividend to shareholders) and $\eta_{2}$ is negative (e.g. through investment in new lines of business), or equivalently $\bar{\eta}=\left(\gamma,-\sqrt{1-\gamma^{2}}\right)$ for some $\gamma \in[0,1]$. In this paper, we establish that the proposed framework includes the model of Gerber and Shiu (2003) as a particular case. Note that our approach differs from Leland (1994), where the author also proposes to model the asset value of a firm by means of a diffusion process and where he examines the optimal capital structure and corporate debts as contingent claims on the firm asset value. The results of Leland (1994) rely on the assumption that the firm asset value is traded (or can be replicated) and extends the results of Black and Cox (1976) to incorporate bankruptcy costs and taxes. The framework exposed in Leland (1994) or Black and Cox (1976) is not applicable to the present situation as the controlled asset firm obeys a stochastic differential equation with a degenerated drift proportional to the local time of the firm on an optimal edge $e(\lambda)$, and as we can no longer rely on the existence of an equivalent risk-neutral measure to price contingent claims on the controlled firm asset value.

The default event is the first hitting time of the firm value $X$ on the edge $e\left(\lambda_{1}\right)$, $\tau=\inf \left\{t \geq 0: X(t) \in e\left(\lambda_{1}\right)\right\}$, with $\inf \varnothing=+\infty$, and the total (discounted) cash flow until ruin time is

$$
J_{\bar{x}}(C(.))=\int_{0}^{\tau} e^{-r s} \sum_{i=1}^{2}\left|\eta_{i}\right| d C(s) .
$$

The constant $r$ is the exogenously given valuation force of interest. It can be for instance the return on a portfolio of long dated (or nowadays even perpetual) fixed income instruments. The force of interest can include a premium for various risks including long term credit, liquidity and reinvestment risk. Hence $r$ can be significantly higher than the money-market spot rate of the Black-Scholes risk-neutral world. The cash flow variable $J_{\bar{x}}(C()$.$) is the total value creation achieved by the ALM strategy as a growth of the$ liabilities or as dividend pay-out to the shareholders. The ALM strategy is fully determined by the choice of $\left\{\bar{\eta}, \lambda_{1}\right\}$. Provided a unit vector $\bar{\eta} \in \mathbb{R}^{2}$, the objective of the ALM committee is the dynamic optimization of the average total discounted creation of value

$$
\begin{aligned}
\tilde{V}(\bar{x}) & =\sup _{C(.)} E\left[J_{\bar{x}}(C(.))\right] \\
& =E\left[J_{\bar{x}}\left(C^{*}(.)\right)\right]
\end{aligned}
$$


over all admissible control functions $C($.$) . Note that a control function C($.$) is called$ admissible, if for every deterministic initial condition, equation (2) has a unique solution which is also weakly unique.

In the sequel, we assume that $r>\mu_{1}>\mu_{2}$, in order to ensure the boundedness of the quantity $\tilde{V}(\bar{x})$, but we will also comment on the case where this assumption is not satisfied. Note that although the assumption $r>\mu_{1}>\mu_{2}$ is only realistic for previsible business lines, it includes some of the most traditional insurance activities as for instance retail life insurance.

In this paper, we derive a two-dimensional Hamilton-Jacobi-Bellman (HJB) optimality equation for $\tilde{V}(\bar{x})$ using singular stochastic control. We show that the optimal control is proportional to the local time of the firm value on the edge $e(\lambda)$ for some constant $\lambda>\lambda_{1}$. The optimal controlled firm value satisfies $X^{*}(t)=X(t \wedge \tau)$ where $X$ is a twodimensional geometric Brownian motion $\eta$-reflected on the edge $e(\lambda)$. More precisely, the process behaves like a geometric Brownian motion in the half-plane below $e(\lambda)$, but at the edge $e(\lambda)$ it is reflected in the specified reflection direction of the vector $\bar{\eta}$. Under the optimal ALM policy, for the particular case $\bar{\eta}=(1,0)$ or $\bar{\eta}=(0,-1)$, we provide a series expansion for the term structure of survival probabilities

$$
P_{\bar{x}}(t):=\mathcal{P}_{\bar{x}}(\tau>t)
$$

as well as for the optimal value creation until time $t$

$$
V(\bar{x}, t)=E_{\bar{x}}\left[\int_{0}^{t \wedge \tau} e^{-r s} \sum_{i=1}^{2}\left|\eta_{i}\right| d C^{*}(s)\right] .
$$

The assumption $r>\mu_{1}>\mu_{2}$ is only needed to ensure the existence of an optimal value of $\lambda$. The expression for the total value creation and the series expansions proposed in the paper remain valid for sub-optimal value $\lambda$ in the case $\mu_{1}>r>\mu_{2}$ as in the original paper of Gerber and Shiu (2003).

The rest of the paper is organized as follows. We start by deriving the HJB equation for $\tilde{V}(\bar{x})$ and we represent the solution in terms of local time. In section 3, we provide an analytical expression to the HJB equation. The result is numerically illustrated and interpreted. In section 4, we study the convergence of $V(\bar{x}, t)$ to $\tilde{V}(\bar{x})$ and we provide series expansions for the probabilities of default using spectral decomposition in the particular cases $\bar{\eta}=(1,0)$ (the ALM committee acts on the assets) and $\bar{\eta}=(0,-1)$ (the ALM committee acts on the liabilities). All the proofs are contained in the Appendix.

\section{Hamilton-Jacobi-Bellman equation for $\tilde{V}(\bar{x})$}

In order to show the optimality of the results in Gerber and Shiu (2003), we use similar arguments as in Harrison and Taksar (1983), Asmussen and Taksar (1997) or Hojgaard and Taksar (1999). As for the one-dimensional case, the Hamilton-JacobiBellman dynamic principle leads to an optimality equation for the expected maximal cash flow $\tilde{V}(\bar{x})$. For a rigorous and complete account on (multi-dimensional) singular stochastic control, we refer to Section VIII in Fleming and Soner (1993). However, similarly to 
Asmussen and Taksar (1997), we give some heuristic arguments that help to interpret the two-dimensional HJB equation. Assume the control process admits the cumulative representation $C(t)=\int_{0}^{t} a(s) d s$ where $a($.$) is non-anticipating with respect to X$ and bounded, or $0 \leq a(t) \leq \theta$ for some constant $\theta<+\infty$. Consider the sub-optimal policy which pays $\left(\left|\eta_{1}\right|+\left|\eta_{2}\right|\right) \alpha$ for some constant $\alpha$ until time $\delta$ or time $\tau$ whenever ruin occurs before $\delta$, and then switch to the optimal strategy $a_{X(\delta)}^{*}(t-\delta)$ :

$$
a(t)= \begin{cases}\alpha, & 0 \leq t \leq \delta \\ a_{X(\delta)}^{*}(t-\delta), & t>\delta .\end{cases}
$$

In view of the sub-optimality of the policy $a($.$) , we deduce the inequality$

$$
\begin{aligned}
\tilde{V}(\bar{x}) & \geq \alpha \delta\left(\left|\eta_{1}\right|+\left|\eta_{2}\right|\right) \mathcal{P}_{\bar{x}}(\tau>\delta)+e^{-r \delta} E_{\bar{x}} \tilde{V}(X(\delta)) \\
& \geq \alpha \delta\left(\left|\eta_{1}\right|+\left|\eta_{2}\right|\right) \mathcal{P}_{\bar{x}}(\tau>\delta)+(1-r \delta) E_{\bar{x}} \tilde{V}(X(\delta))
\end{aligned}
$$

As $\mathcal{P}_{\bar{x}}(\tau>\delta) \approx 1$ for small $\delta$ and with the assumption that the optimal gain function $\tilde{V}(\bar{x})$ is twice-differentiable, by standard application of multi-dimensional Itô Lemma to $\tilde{V}(X(\delta))$ and dividing by $\delta$, we finally obtain

$$
\mathcal{G} \tilde{V}(\bar{x})-r \tilde{V}(\bar{x})-\alpha\left(\left|\eta_{1}\right|+\left|\eta_{2}\right|-\nabla_{\eta} \tilde{V}(\bar{x})\right) \leq 0,
$$

with $\mathcal{G}$ the infinitesimal generator of the bivariate geometric Brownian motion

$$
\mathcal{G}=\frac{1}{2} \sum_{i, j=1}^{2} \sigma_{i, j}^{2} x_{i} x_{j} \frac{\partial^{2}}{\partial x_{i} \partial x_{j}}+\sum_{i=1}^{2} \mu_{i} x_{i} \frac{\partial}{\partial x_{i}} .
$$

It can be shown that this inequality is binding for at least one $\alpha \in[0, \theta]$, see Fleming and Soner (1993). As the left-hand-side of equation (3) is linear in $\alpha$ provided $\bar{x}$, the maximum is attained either for $\alpha=0$ or $\alpha=\theta$. It is then obvious that the optimal control satisfies

$$
\alpha(\bar{x})=\theta 1_{A}(\bar{x}) \quad \text { where } \quad A=\left\{\bar{x} \in \mathbb{R}^{2}: \nabla_{\eta} \tilde{V}(\bar{x}) \geq\left|\eta_{1}\right|+\left|\eta_{2}\right|\right\} .
$$

Such strategy is called a Bang-Bang strategy, namely paying the maximum or no control is applied.

Let the upper limit $\theta$ go to infinity. The unboundedness of the control variable $\alpha($. suggests that either a degenerated impulse (paying the maximum) pushes the firm value to below along the direction indicated by the vector $\bar{\eta}$ or no control is applied until the firm value exits at small surrounding interval. Under the first option, the total expected cash flow can be decomposed as

$$
\left(\left|\eta_{1}\right|+\left|\eta_{2}\right|\right) \epsilon+\tilde{V}\left(x_{1}-\eta_{1} \epsilon, x_{2}-\eta_{2} \epsilon\right) \approx \tilde{V}(\bar{x})+\left(\left|\eta_{1}\right|+\left|\eta_{2}\right|-\nabla_{\eta} \tilde{V}(\bar{x})\right) \epsilon
$$

and under the second one it is

$$
\begin{aligned}
E_{\bar{x}}\left[e^{-r \tau(\epsilon)} \tilde{V}(X(\tau(\epsilon)))\right] & \approx \tilde{V}(\bar{x})+(\mathcal{G} \tilde{V}(\bar{x})-r \tilde{V}(\bar{x})) E_{\bar{x}}[\tau(\epsilon)] \\
& \approx \tilde{V}(\bar{x})+(\mathcal{G} \tilde{V}(\bar{x})-r \tilde{V}(\bar{x})) \epsilon
\end{aligned}
$$


where $\tau(\epsilon)=\inf \{t \geq 0:\|X(t)-\bar{x}\|=\epsilon\}$. As we choose the maximum of both alternatives, we obtain the optimality equation

$$
\tilde{V}(\bar{x})=\max \left\{\tilde{V}(\bar{x})+(\mathcal{G} \tilde{V}(\bar{x})-r \tilde{V}(\bar{x})) \epsilon, \tilde{V}(\bar{x})+\left(\left(\left|\eta_{1}\right|+\left|\eta_{2}\right|\right)-\nabla_{\eta} \tilde{V}(\bar{x})\right) \epsilon\right\} .
$$

Subtracting both sides of the previous relation by $\tilde{V}(\bar{x})$ leads intuitively to the following singular HJB equation.

Theorem 1 Assume the optimal function $\tilde{V}(\bar{x})$ is twice continuous differentiable. Then the optimal function $\tilde{V}(\bar{x})$ satisfies the following Hamilton-Jacobi-Bellman equation

$$
\max \left\{\mathcal{G} \tilde{V}(\bar{x})-r \tilde{V}(\bar{x}), \sum_{i=1}^{2}\left|\eta_{i}\right|-\nabla_{\eta} \tilde{V}(\bar{x})\right\}=0,
$$

with $\tilde{V}(\bar{x})=0 \quad \forall \bar{x} \in e\left(\lambda_{1}\right)$, where $r$ is the discount factor, $\mathcal{G}$ is the infinitesimal generator of the bivariate geometric Brownian motion as in (4), and $\nabla_{\eta} \tilde{V}(\bar{x})=\sum_{i=1}^{2} \eta_{i} \frac{\partial \tilde{V}}{\partial x_{i}}(\bar{x})$ is the directional derivative along the unit reflection vector $\eta$.

We can further describe the optimal function $\tilde{V}(\bar{x})$. It can be shown that $\tilde{V}(\bar{x})$ is concave with $\tilde{V}(\bar{x}) \rightarrow+\infty$ as $\left|x_{i}\right| \rightarrow+\infty$. Moreover, the control $\alpha C($.$) is admissible for$ the initial firm value $\alpha \bar{x}$ whenever the control $C($.$) is admissible for the initial firm value$ $\bar{x}$ and $J(\alpha C())=.\alpha J(C()$.$) . We conclude that \tilde{V}(\bar{x})$ is homogeneous of degree 1 in its argument, $\tilde{V}(\alpha \bar{x})=\alpha \tilde{V}(\bar{x})$, and thus

$$
\nabla_{\eta} \tilde{V}(\alpha \bar{x})=\nabla_{\eta} \tilde{V}(\bar{x}) .
$$

The concavity of $\tilde{V}(\bar{x})$ together with the scaling property $\nabla_{\eta} \tilde{V}(\alpha \bar{x})=\nabla_{\eta} \tilde{V}(\bar{x})$ yield that the set $A$ defined by equation (5) is the half-plane

$$
A=\left\{\bar{x}=\left(x_{1}, x_{2}\right) \in \mathbb{R}^{2}: x_{1} \geq \lambda x_{2}\right\}
$$

for some constant $\lambda>0$. Let $O$ be the optimal set defined in the introduction

$$
O=\left\{\bar{x}=\left(x_{1}, x_{2}\right) \in \mathbb{R}^{2}: x_{1} \leq \lambda x_{2} \text { and } x_{1}>\lambda_{1} x_{2}\right\} .
$$

The above considerations and the HJB equation yield that $(\mathcal{G}-r) \tilde{V}(\bar{x})=0$ and $\nabla_{\eta} \tilde{V}(\bar{x}) \geq$ $\sum_{i=1}^{2}\left|\eta_{i}\right|$ on $O$. It implies that the optimal control is the local time of $X^{*}(t)=X(t \wedge \tau)$ on the (optimal) edge $e(\lambda)$ and $X$ is a bivariate geometric Brownian $\eta$-reflected on $e(\lambda)$. The proof is based on multi-dimensional singular stochastic control and is provided in the Appendix.

Theorem 2 (Verification theorem) For any admissible control $C($.$) , we have$

$$
\tilde{V}(\bar{x}) \geq E J_{\bar{x}}(C(.)) \text {. }
$$

Moreover, the optimal control is the local time of the process $X^{*}$ on $e(\lambda), C^{*}(t)=$ $L_{t}^{e(\lambda)}\left(X^{*}\right)$, and the controlled firm value is a bivariate geometric Brownian motion instantaneously $\eta$-reflected on the edge $e(\lambda)$ and instantaneously killed on the edge $e\left(\lambda_{1}\right)$. 
In this paper, we also investigate the optimal value creation $V(\bar{x}, t)$ until time $t$ whenever ruin time occurs after $t$

$$
V(\bar{x}, t)=E_{\bar{x}}\left[\int_{0}^{t \wedge \tau} e^{-r s} \sum_{i=1}^{2}\left|\eta_{i}\right| d L_{s}^{e(\lambda)}(X)\right] .
$$

To start with, the moment generating function of the quantity $\int_{0}^{t \wedge \tau} \sum_{i=1}^{2}\left|\eta_{i}\right| d L_{s}^{e(\lambda)}(X)$ satisfies the two-dimensional partial differential equation given in the next proposition. The proof uses similar arguments as in Burdzy et al. (2004) and is given in the Appendix.

Proposition 1 Let $X$ be a bivariate geometric BM with drift $\mu$ started at $\bar{x}$ (in the half-plane below $e(\lambda)$ ), $\eta$-reflected on $e(\lambda)$ and killed at his first visit on the lower edge $e\left(\lambda_{1}\right)$. Suppose the twice-differentiable function $I(\bar{x}, t)$ is a smooth solution to the partial differential equation

$$
\frac{\partial}{\partial t} I(\bar{x}, t)=\mathcal{G} I(\bar{x}, t), \quad I(\bar{x}, 0)=1
$$

with $\mathcal{G}$ the infinitesimal generator of the bivariate geometric Brownian motion as in (4), subject to the elastic boundary condition $\nabla_{\eta} I(\bar{x}, t)=\alpha \sum_{i=1}^{2}\left|\eta_{i}\right| I(\bar{x}, t)$ for all $x \in e(\lambda)$ and $I(\bar{x}, t)=1$ for all $\bar{x} \in e\left(\lambda_{1}\right)$. Then the following representation holds

$$
I(\bar{x}, t)=E_{\bar{x}}\left[e^{\alpha \int_{0}^{t \wedge \tau} \sum_{i=1}^{2}\left|\eta_{i}\right| d L_{s}^{e(\lambda)}(X)}\right]
$$

where $L_{s}^{e(\lambda)}(X)$ is the local time of $X$ on $e(\lambda)$.

A series expansion of the exponential function in equation (8) leads to a partial differential equation for the moments $K_{n}(\bar{x}, t)$ of the quantity $\int_{0}^{t \wedge \tau} \sum_{i=1}^{2}\left|\eta_{i}\right| d L_{s}^{e(\lambda)}(X)$

$$
K_{n}(\bar{x}, t)=E_{\bar{x}}\left[\left(\sum_{i=1}^{2}\left|\eta_{i}\right| \int_{0}^{\tau \wedge t} d L_{s}^{e(\lambda)}(X)\right)^{n}\right] .
$$

Inserting the following expansion

$$
E_{\bar{x}}\left[e^{\alpha \int_{0}^{\tau \wedge t} \sum_{i=1}^{2}\left|\eta_{i}\right| d L_{s}^{e(\lambda)}(X)}\right]=\sum_{n=0}^{+\infty} \frac{\alpha^{n}}{n !} K_{n}(\bar{x}, t),
$$

in the partial differential equation of Proposition 1 and comparing the term of same order in $\alpha$, we finally obtain

$$
\frac{\partial}{\partial t} K_{n}(\bar{x}, t)=\mathcal{G} K_{n}(\bar{x}, t), \quad \forall n \in \mathcal{N}
$$

together with the boundary conditions $K_{n}(\bar{x}, t)=0$ for all $x \in e\left(\lambda_{1}\right)$, and

$$
\nabla_{\eta} K_{1}(\bar{x}, t)=\sum_{i=1}^{2}\left|\eta_{i}\right|, \quad \nabla_{\eta} K_{n}(\bar{x}, t)=n \sum_{i=1}^{2}\left|\eta_{i}\right| K_{n-1}(\bar{x}, t), \quad n \neq 1
$$

for all $\bar{x} \in e(\lambda)$. As the optimal value creation $V(\bar{x}, t)$ until time $t$ is precisely equal to $\int_{0}^{t} e^{-r s} d K_{1}(\bar{x}, s)$, it satisfies the following partial differential equation

$$
\frac{\partial}{\partial t} V(\bar{x}, t)=(\mathcal{G}-r) V(\bar{x}, t)
$$


subject to the same boundary conditions as $K_{1}(\bar{x}, t)$. In what follows, we are able to solve the partial differential equation (10) for the particular cases $\bar{\eta}=(1,0)$ (the ALM committee acts on the assets) and $\bar{\eta}=(0,-1)$ (the ALM committee acts on the liabilities).

\section{Solving the Hamilton-Jacobi-Bellman equation}

In this section, we provide analytical solutions to the HJB equation for the optimal value creation $\tilde{V}(\bar{x})$. The results are graphically illustrated and interpreted. As suggested by Davis and Norman (1990) in the context of portfolio selection with transaction costs, the two-dimensional HJB optimality equation can be simplified using the trivial scaling property $\tilde{V}(\alpha \bar{x})=\alpha \tilde{V}(\bar{x})$ or equivalently

$$
\tilde{V}\left(x_{1}, x_{2}\right)=\left(x_{1}+x_{2}\right) \tilde{V}\left(\frac{x_{1}}{x_{1}+x_{2}}, 1-\frac{x_{1}}{x_{1}+x_{2}}\right)=:\left(x_{1}+x_{2}\right) \tilde{W}(q), \quad q=\frac{x_{1}}{x_{1}+x_{2}} .
$$

It leads to an analytical expression for the optimal value creation given the vector $\bar{\eta}=$ $\left(\eta_{1}, \eta_{2}\right)$. The next proposition extends the results obtained in Gerber and Shiu (2003) to the more general situation allowing both the control of the liability and the asset values of the firm. The condition $r>\mu_{1}>\mu_{2}$ is assumed but the analytical expression provided for $\tilde{V}(\bar{x})$ remains valid in the case $\mu_{1}>r>\mu_{2}$ for a fixed sub-optimal value of $\lambda>\lambda_{1}$. The details of the proof can be found in the Appendix.

Proposition 2 Consider the indicial equation

$$
\frac{\sigma^{2}}{2} \alpha(\alpha-1)+\left(\mu_{1}-\mu_{2}\right) \alpha+\mu_{2}-r=0
$$

where $\sigma^{2}=\sigma_{1}^{2}+\sigma_{2}^{2}-2 \rho \sigma_{1} \sigma_{2}$ and $r>\mu_{1}>\mu_{2}$, with solutions $\alpha_{1}<0$ and $\alpha_{2}>1$. The optimal average discounted cash flow $\tilde{V}(\bar{x})$ for $\bar{x}$ in $O$ is given by

$$
\tilde{V}\left(x_{1}, x_{2}\right)=\frac{\left(\left|\eta_{1}\right|+\left|\eta_{2}\right|\right)\left(\lambda_{1}^{\alpha_{2}} x_{1}^{\alpha_{1}} x_{2}^{1-\alpha_{1}}-\lambda_{1}^{\alpha_{1}} x_{1}^{\alpha_{2}} x_{2}^{1-\alpha_{2}}\right)}{\eta_{1}\left(\alpha_{1} \lambda^{\alpha_{1}-1} \lambda_{1}^{\alpha_{2}}-\alpha_{2} \lambda_{1}^{\alpha_{1}} \lambda^{\alpha_{2}-1}\right)+\eta_{2}\left(\left(1-\alpha_{1}\right) \lambda^{\alpha_{1}} \lambda_{1}^{\alpha_{2}}-\left(1-\alpha_{2}\right) \lambda_{1}^{\alpha_{1}} \lambda^{\alpha_{2}}\right)}
$$

and the optimal $\lambda_{*}>\lambda_{1}$ is given by

$$
\lambda_{*}=\left(\frac{\alpha_{2}\left(\alpha_{2}-1\right)}{\alpha_{1}\left(\alpha_{1}-1\right)}\right)^{\frac{1}{\alpha_{1}-\alpha_{2}}} \cdot \lambda_{1}
$$

whatever the reflection vector $\bar{\eta}$.

The conditions $r>\mu_{2}$ and $\mu_{1}>\mu_{2}$ guarantee the existence of two distinct real solutions $\alpha_{1}$ and $\alpha_{2}$ (with $\alpha_{2}>\alpha_{1}$ and $\alpha_{1}<0$ ) to the indicial ordinary differential equation (11). In the case $r>\mu_{1}$, the inequality $\alpha_{2}>1$ ensures the existence of an optimal value for $\lambda$. When $\mu_{1}$ converges to $r$, the solution $\alpha_{2}$ tends to 1 and, as a consequence, $\lambda_{*}$ goes to $+\infty$. In the limiting case $\mu_{1}=r$, it is optimal never to pay dividends nor reinvest in the firm activities $\left(\lambda_{*}=+\infty\right)$, but the optimal value $\tilde{V}(\bar{x})$ creation is finite and is equal to

$$
\tilde{V}\left(x_{1}, x_{2}\right)=\frac{\left(\left|\eta_{1}\right|+\left|\eta_{2}\right|\right)}{\eta_{1}} x_{1}\left(1-x_{1}^{\alpha_{1}-1}\left(\lambda_{1} x_{2}\right)^{1-\alpha_{1}}\right) .
$$


In the case $\mu_{1}>r$, there is no optimal value for $\lambda$ and the (non optimal) value creation $\tilde{V}(\bar{x})$ goes to $+\infty$ when $\lambda$ tends to $+\infty$. We can interpret the latter observation as the asset growth rate being so high that it is more profitable to keep the firm asset unchanged than to pay dividends or to reinvest in the firm activities. Remark that in that case the formula provided for the value creation remains valid for exogenously given (non optimal) value of $\lambda$. Finally, in the limit case $\mu_{1}=\mu_{2}$, it is trivial to check that $\alpha_{1}+\alpha_{2}=1$ and as a consequence $\lambda_{*}=\lambda_{1}$. In that situation, it is more profitable never to start the business and declare bankruptcy.

The situation $r>\mu_{1}>\mu_{2}$ of Proposition 2 is the most interesting case from a theoretical point of view because both $\lambda_{*}$ and $\tilde{V}(\bar{x})$ are finite. Figure 1 plots the optimal $\lambda_{*}$ as a function of the volatility $\sigma$ and the return on assets $\mu_{1}$ provided the growth rate of liabilities $\mu_{2}=0.04$ and the risk-free interest rate $r=0.08$. We observe that the optimal $\lambda$ increases both with the volatility and with the return on assets. Moreover, the value of $\lambda_{*}$ increases faster with $\mu_{1}$ for higher values of the volatility $\sigma$. Hence if the risk is high compared to the potential return, it is better to start paying dividends and investing into new lines of business later and reduce consequently the risk of early bankruptcy. On the other hand, if the return is high compared to the volatility, it is optimal to pay dividends in an earlier phase of the business cycle. Remark that Hojgaard and Taksar (1999) draw similar conclusions.

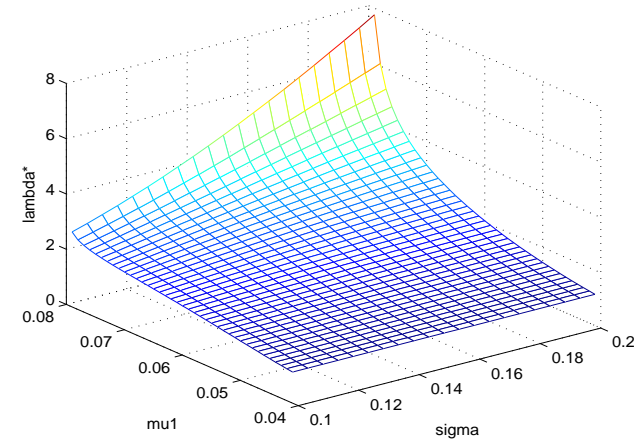

Fig. 1. Optimal value of $\lambda$ as a function of $\mu_{1}$ and $\sigma$ provided $\mu_{2}=0.04$ and $r=0.08$.

It is interesting to look at the dependence of $\tilde{V}(\bar{x})$ on the reflection vector $\bar{\eta}$. From expression (12), it follows that there exists a value $\lambda_{1}^{*}$ for the ruin limit such that the optimal value creation is independent of the reflection vector $\bar{\eta}$. In view of the expression for $\tilde{V}(\bar{x})$ as given in (12), this particular value of the solvency limit satisfies

$$
\left(\alpha_{2} \lambda_{1}^{\alpha_{1}} \lambda^{\alpha_{2}-1}-\alpha_{1} \lambda^{\alpha_{1}-1} \lambda_{1}^{\alpha_{2}}\right)=\left(\left(1-\alpha_{1}\right) \lambda^{\alpha_{1}} \lambda_{1}^{\alpha_{2}}-\left(1-\alpha_{2}\right) \lambda_{1}^{\alpha_{1}} \lambda^{\alpha_{2}}\right),
$$

or equivalently

$$
\lambda_{1}^{*}=\left(\frac{\alpha_{1} \lambda^{\alpha_{1}-1}-\left(\alpha_{1}-1\right) \lambda^{\alpha_{1}}}{\alpha_{2} \lambda^{\alpha_{2}-1}-\left(\alpha_{2}-1\right) \lambda^{\alpha_{2}}}\right)^{\frac{1}{\alpha_{1}-\alpha_{2}}} .
$$

If the condition $\mu_{1}>\mu_{2}$ is satisfied, this implies that $\lambda_{1}^{*}>1$. Moreover, when $\mu_{2}$ tends to $\mu_{1}, \lambda_{*}$ approaches $\lambda_{1}$ and as a consequence, $\lambda_{1}^{*}$ goes to 1 . 
Figures 2 and 3 compare the total value creation $\tilde{V}(\bar{x})$ at the optimal edge $e(\lambda)$ for different bankruptcy limits $\lambda_{1}$ as a function of $\gamma$ defined such that $\bar{\eta}=\left(\gamma,-\sqrt{1-\gamma^{2}}\right)$. The starting liability value $x_{2}$ of the firm is normalized to 1 . The total value creation is an increasing function of $\gamma$ if $\lambda_{1}<\lambda_{1}^{*}$; it is a decreasing function if $\lambda_{1}>\lambda_{1}^{*}$. An interpretation of this observation is the following. If the liability and asset growth rates are close to each other, it is optimal to raise the liabilities rather than to pay dividends when the regulator allows for temporary deficit $\left(\lambda_{1}<\lambda_{1}^{*} \approx 1\right)$. On the other hand, the ALM committee will prefer to pay dividends if the regulator requires economic capital $\left(\lambda_{1}>\lambda_{1}^{*} \approx 1\right)$. However, if $\mu_{1}$ is significantly larger than $\mu_{2}$, it can be more profitable to raise the liabilities rather than to pay dividends and reduce consequently the asset firm value even for values of $\lambda_{1}$ slightly higher than 1 . We conclude that either for $\gamma=1$ or for $\gamma=0$ the total value creation is maximized. This result justifies the choice to pay more attention to the particular cases $\bar{\eta}=(1,0)$ and $\bar{\eta}=(0,-1)$.

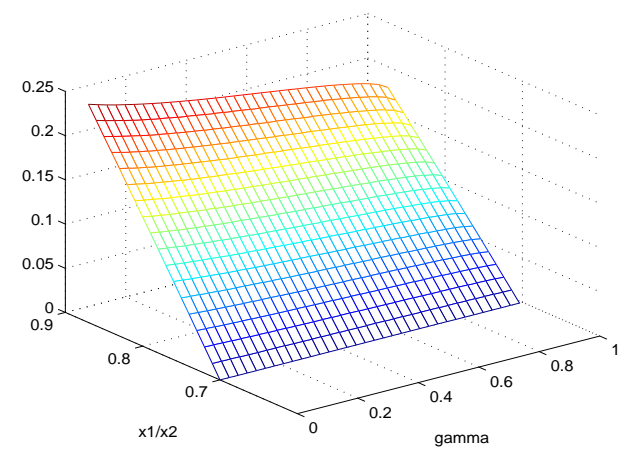

Fig. 2. Total value creation on the optimal edge for $\mu_{1}=0.06, \mu_{2}=0.055, \sigma_{1}=\sigma_{2}=0.15, \rho=0.8$ and $\lambda_{1}=0.7$.

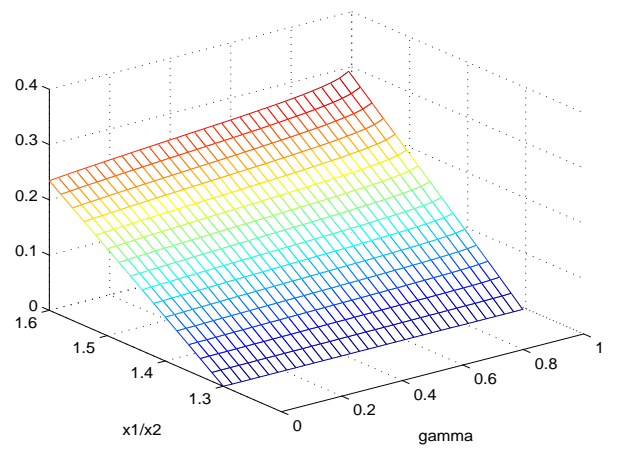

Fig. 3. Total value creation on the optimal edge for $\mu_{1}=0.06, \mu_{2}=0.055, \sigma_{1}=\sigma_{2}=0.15, \rho=0.8$ and $\lambda_{1}=1.3$. 


\section{Spectral decomposition}

In the previous sections, we proved that the optimal cash flow is proportional to the local time of the controlled firm value $X^{*}(t)=X(t \wedge \tau)$ along the edge $e\left(\lambda_{*}\right)$ for some constant $\lambda_{*}>\lambda_{1}$, when the process $X$ is a geometric Brownian motion $\eta$-reflected on $e\left(\lambda_{*}\right)$. In this section, as suggested in Gerber and Shiu (2003) we study the convergence of the average cash flow $V(\bar{x}, t)$ accumulated up to time $t$ whenever ruin time occurs after $t$

$$
V(\bar{x}, t)=E_{\bar{x}}\left[\int_{0}^{\tau \wedge t} e^{-r s} d L_{s}^{e\left(\lambda_{*}\right)}(X)\right]
$$

to the stationary value creation $\tilde{V}(\bar{x})$. In the general case, one needs to solve numerically the partial differential equation

$$
\frac{\partial}{\partial t} V(\bar{x}, t)=(\mathcal{G}-r) V(\bar{x}, t)
$$

derived in Proposition 1. Note that the probability of ruin can also be found as the solution to the same partial differential equation with $r=0$ and suitable initial condition. Traditional methods to solve equation (14) include finite-difference or Monte-Carlo simulation.

Our approach is different, as we will look at spectral representations, or eigendifferential expansions as they are called in Itô and McKean (1965). This technique was already used for financial issues earlier, e.g. by Lewis (1998) or Linetsky (2004a, 2004b, 2004c), but the integration of this concept into discounted cash-flows in asset-liability management is new. Spectral expansions are of interest for several reasons. They allow the investigation of the convergence of the discounted cash flows together with the time-evolution of the ruin probability, which leads to a finer understanding of the risks involved in an assetliability management policy. The first eigenvalue in the expansion has also a specific financial meaning, as pointed out by Linetsky, in the context of term-structure modeling. We will come back to this when we discuss the resulting expansions.

For our investigation here, the use of spectral theory in particular offers a tractable alternative for the special cases $\bar{\eta}=(1,0)$ or $\bar{\eta}=(0,-1)$. In general, the domain of the two-dimensional firm value process is not bounded, and a spectral expansion of $V(\bar{x}, t)$ is not trivial as the spectrum is not guaranteed to be discrete and of multiplicity one. However, in the particular situations $\bar{\eta}=(1,0)$ and $\bar{\eta}=(0,-1)$, the problem can be reduced to a scalar spectral decomposition on a bounded domain with discrete spectrum. In what follows, we assume that the ALM committee only acts on the assets, or $\bar{\eta}=(1,0)$. We are confident that the interested reader will easily extend the results to the case $\bar{\eta}=$ $(0,-1)$. Moreover, for the sake of simplicity and without loss of generality, we normalize the liability value at time 0 (notation $x_{2}$ ) to 1 . We provide a series expansion for $V(\bar{x}, t)$ as well as for the survival probability $P_{\bar{x}}(t):=\mathcal{P}_{\bar{x}}(\tau>t)$. As we consider the case $\bar{\eta}=(1,0)$, it is convenient to apply the following transformation

$$
\begin{aligned}
Y_{1} & =\frac{X_{1}}{X_{2}} \\
Y_{2} & =X_{2} .
\end{aligned}
$$


The bivariate process $Y=\left(Y_{1}, Y_{2}\right)$ remains a two-dimensional reflecting geometric Brownian motion whose generator is

$$
\hat{\mathcal{G}}=\frac{1}{2} \sum_{i, j=1}^{2} \hat{\sigma}_{i, j}^{2} y_{i} y_{j} \frac{\partial^{2}}{\partial y_{i} \partial y_{j}}+\sum_{i=1}^{2} \hat{\mu}_{i} y_{i} \frac{\partial}{\partial y_{i}}
$$

with drift $\hat{\mu}=\left(\left(\mu_{1}-\mu_{2}\right)+\sigma_{2}^{2}-\rho \sigma_{1} \sigma_{2}, \mu_{2}\right)$ and covariance matrix

$$
\left(\hat{\sigma}_{i, j}^{2}\right)=\left(\begin{array}{cc}
\sigma^{2} & \rho \sigma_{1} \sigma_{2}-\sigma_{2}^{2} \\
\rho \sigma_{1} \sigma_{2}-\sigma_{2}^{2} & \sigma_{2}^{2}
\end{array}\right) .
$$

In the coordinates (15), the set $O$ is transformed into

$$
\hat{O}=\left\{\bar{y}=\left(y_{1}, y_{2}\right) \in \mathbb{R}^{2}: \lambda_{1}<y_{1} \leq \lambda\right\} .
$$

Solving the partial differential equation (14) in the coordinates (15) simplifies to a scalar diffusion problem as the accumulated value creation is a homogeneous function and as the survival probability $P_{\bar{x}}(t)$ only depends on $y_{1}$. It leads to the series expansions given in the next propositions; the details of the proof can be found in the Appendix.

Proposition 3 The survival probabilities have the following spectral decomposition

$$
\mathcal{P}_{\bar{x}}(\tau>t)=\sum_{n=0}^{+\infty} c_{n} e^{-E_{n}^{P} t} \phi_{n}(z), \quad z=x_{1} / x_{2}
$$

with eigenfunctions

$$
\phi_{n}(z)=z^{\theta} \cos (b \ln z)+c z^{\theta} \sin (b \ln z),
$$

where $\theta=\frac{1}{2}(1-\alpha), b=\frac{1}{2} \sqrt{4 \beta-(1-\alpha)^{2}}, \alpha=\frac{2 \hat{\mu}_{1}}{\sigma^{2}}, \beta=\frac{2 E_{n}^{P}}{\sigma^{2}}$ and $c=-\operatorname{cotg}\left(b \ln \lambda_{1}\right)$. The eigenvalues $\left\{E_{n}^{P}, n \in \mathcal{N}\right\}$ are the solutions to

$$
\theta(\cos (b \ln \lambda)+c \sin (b \ln \lambda))+b(c \cos (b \ln \lambda)-\sin (b \ln \lambda))=0,
$$

and the coefficients $c_{n}$ satisfy

$$
c_{n}=\frac{\int_{\lambda_{1}}^{\lambda} \phi_{n}(z) w(z) d z}{\int_{\lambda_{1}}^{\lambda} \phi_{n}^{2}(z) w(z) d z}
$$

where $w(z)=\frac{2 z^{\gamma-2}}{\sigma^{2}}$ and $\gamma=\frac{2 \hat{\mu}_{1}}{\sigma^{2}}$.

Proposition 4 The function $W(z, t) \equiv V\left(\left(\frac{x_{1}}{x_{2}}, 1\right), t\right)$ (with $\left.z=x_{1} / x_{2}\right)$ has the following spectral decomposition on the interval $\left(\lambda_{1}, \lambda\right]$

$$
W(z, t)=\breve{V}(z)-\sum_{n=1}^{+\infty} c_{n} e^{-E_{n}^{V} t} \phi_{n}(z), \quad z=x_{1} / x_{2} \in\left(\lambda_{1}, \lambda\right]
$$

with $\breve{V}(z) \equiv V\left(\left(\frac{x_{1}}{x_{2}}, 1\right)\right)\left(z=x_{1} / x_{2}\right)$ and with eigenfunctions

$$
\phi_{n}(z)=z^{\theta} \cos (b \ln z)+c z^{\theta} \sin (b \ln z),
$$


where $\theta=\frac{1}{2}(1-\alpha), b=\frac{1}{2} \sqrt{4 \beta-(1-\alpha)^{2}}, \alpha=\frac{2\left(\mu_{1}-\mu_{2}\right)}{\sigma^{2}}, \beta=\frac{2\left(\mu_{2}-r+E_{n}^{V}\right)}{\sigma^{2}}$ and $c=$ $-\operatorname{cotg}\left(b \ln \lambda_{1}\right)$. The eigenvalues $\left\{E_{n}^{V}, n \in \mathcal{N}\right\}$ are the solutions to

$$
\theta(\cos (b \ln \lambda)+c \sin (b \ln \lambda))+b(c \cos (b \ln \lambda)-\sin (b \ln \lambda))=0,
$$

and the coefficients $c_{n}$ satisfy

$$
c_{n}=\frac{\int_{\lambda_{1}}^{\lambda} \breve{V}(z) \phi_{n}(z) w(z) d z}{\int_{\lambda_{1}}^{\lambda} \phi_{n}^{2}(z) w(z) d z}
$$

where $w(z)=\frac{2 z^{\gamma-2}}{\sigma^{2}}$ and $\gamma=\frac{2\left(\mu_{1}-\mu_{2}\right)}{\sigma^{2}}$.

Spectral expansions have convergence pattern opposite to more traditional numerical methods as finite-difference solver or Monte-Carlo simulation. For shorter maturities, more terms in the series need to be added to reach the same accuracy. The convergence depends on the model parameters. As the difference $\mu_{1}-\mu_{2}$ increases or $\sigma^{2}$ goes to zero, the discrete spectrum tends to be continuous, the first eigenvalue goes to zero and, as a consequence, the series becomes oscillating. Figure 4 illustrates this hehaviour. In all calculations $r=0.08, \mu_{1}=0.06, \sigma_{1}=\sigma_{2}=0.25, \rho=0.3$ and $\lambda_{1}=0.9$ are fixed. Figure 4 displays the series expansion with 15 terms for the survival probabilities $P_{\bar{x}}(t)$ after 5 years as a function of $z=x_{1} / x_{2}$ for different liability growth rate $\mu_{2}$ and non-optimal value of $\lambda$ fixed to 1.2. The same remark also holds for the spectral decomposition of the value creation.

The first eigenvalue has a nice asymptotic interpretation. For $t$ going to infinity, the survival probability satisfies

$$
E_{0}^{P} \approx-\frac{\partial \ln P_{\bar{x}}(t)}{\partial t}
$$

which means that the first eigenvalue $E_{0}^{P}$ is the asymptotic constant intensity of default. A similar interpretation of the first eigenvalue $E_{0}^{V}$ as an asymptotic relative rate of growth also holds for the total value creation $V(\bar{x}, t)$. In what follows, comparing the first eigenvalues of the value creation and the survival probabilities allows for a deeper understanding of the ALM policy. In figure 5 and 7 we plot the series expansions for $V(z, t)$ with 15 terms as a function of the time and the ratio $x_{1} / x_{2}$. The first eigenvalue $E_{0}^{V}$ is equal to 0.2111 for Figure $5\left(\mu_{1}-\mu_{2}=0.005\right)$ and to 0.0752 for Figure $7\left(\mu_{1}-\mu_{2}=\right.$ $0.01)$. Figure 6 and 8 present the spectral decomposition of the survival probabilities $P_{\bar{x}}(t)$ also with 15 terms and the same sets of model parameters. The first eigenvalue $E_{0}^{P}$ is equal to 0.0567 for Figure 6 and to 0.0037 for Figure 8. The series expansions for both the value creation and the survival probabilities converge faster to their stationary values when $\mu_{2}$ is closer to $\mu_{1}$. This can be perfectly explained. Indeed, when the liability growth rate tends to the asset growth rate, the risk of early bankruptcy increases together with the chance of having achieved almost the asymptotic value creation at ruin time. However, as the first eigenvalue decreases faster with the difference $\mu_{1}-\mu_{2}$ for the survival probabilities than for the value creation, the health of the company deteriorates when $\mu_{2}$ approaches $\mu_{1}$. 


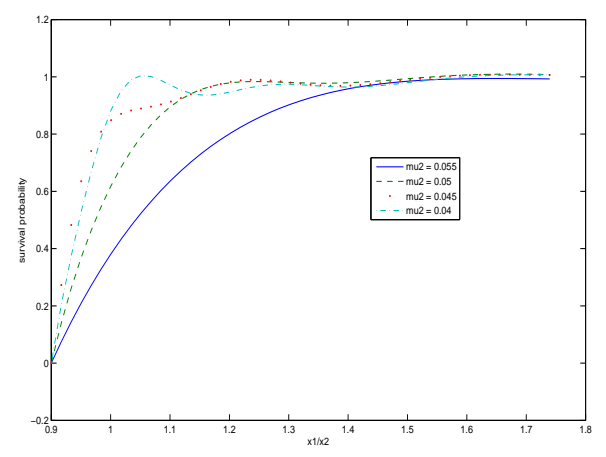

Fig. 4. Convergence pattern for the spectral expansions of the survival probabilities for for $r=0.08$, $\mu_{1}=0.06, \sigma_{1}=\sigma_{2}=0.25, \rho=0.3, \lambda_{1}=0.9$ and $t=5$.

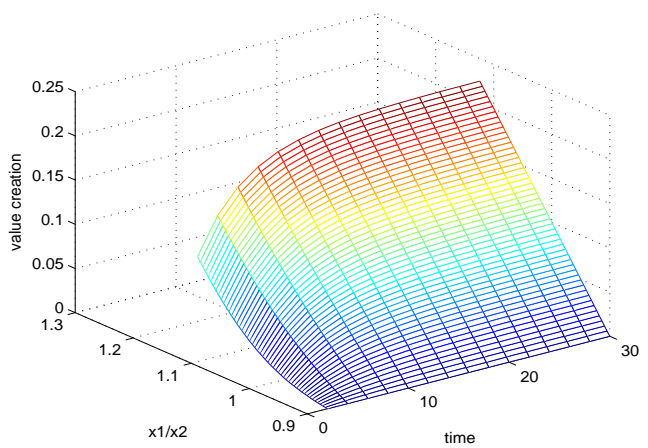

Fig. 5. Convergence of the value creation to the stationary solution $V(\bar{x})$ normalizing $x_{2}$ to 1 for $r=0.08$, $\mu_{1}=0.06, \mu_{2}=0.055, \sigma_{1}=\sigma_{2}=0.25, \rho=0.3$ and $\lambda_{1}=0.9$.

\section{Acknowledgement}

The authors wish to thank the "Onderzoeksraad K.U.Leuven" for a GOA grant. They also thank the referees for their constructive comments, and for helping to improve the contents of this paper.

\section{Appendix A. Appendix}

\section{A.1. Proof of Theorem 2 (Verification theorem)}

We use similar arguments as in Asmussen and Taksar (1997). Consider an admissible control $C($.$) . To start with, we decompose C($.$) into its continuous part C^{c}($.$) and its$ discontinuous part $C^{d}()=.\sum_{0<s<t}\left[C(s)-C\left(s_{-}\right)\right]$. By standard application of the (twodimensional) change of variable formula for semimartingales as e.g. in Asmussen and Taksar (1997), we obtain 


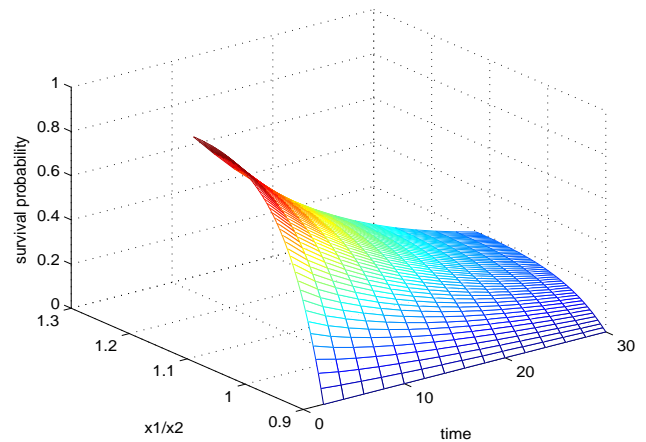

Fig. 6. Convergence of the survival probability for $r=0.08, \mu_{1}=0.06, \mu_{2}=0.055, \sigma_{1}=\sigma_{2}=0.25$, $\rho=0.3$ and $\lambda_{1}=0.9$.

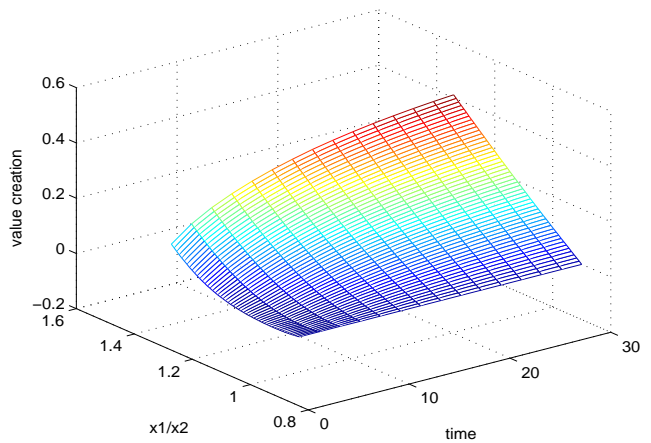

Fig. 7. Convergence of the value creation to the stationary solution $V(\bar{x})$ normalizing $x_{2}$ to 1 for $r=0.08$, $\mu_{1}=0.06, \mu_{2}=0.05, \sigma_{1}=\sigma_{2}=0.25, \rho=0.3$ and $\lambda_{1}=0.9$.

$$
\begin{aligned}
e^{-r(\tau \wedge t) \tilde{V}(X(\tau \wedge t))=} & \tilde{V}(\bar{x})+\int_{0}^{\tau \wedge t} e^{-r s}(\mathcal{G}-r) \tilde{V}(X(s)) d s \\
& +\sum_{i=1}^{2} \int_{0}^{\tau \wedge t} e^{-r s} \frac{\partial \tilde{V}}{\partial x_{i}}(X(s)) \sigma_{i} X_{i}(s) d B_{i}(s) \\
& -\sum_{i=1}^{2} \int_{0}^{\tau \wedge t} e^{-r s} \frac{\partial \tilde{V}}{\partial x_{i}}(X(s)) \eta_{i} d C^{c}(s) \\
& -\sum_{0 \leq s \leq \tau \wedge t} e^{-r s}\left[\tilde{V}(X(s))-\tilde{V}\left(X\left(s_{-}\right)\right)\right]
\end{aligned}
$$

where $\mathcal{G}$ is the infinitesimal generator of the geometric Brownian motion. Since $\tilde{V}(\bar{x})$ satisfies the HJB optimality equation, we have $(\mathcal{G}-r) \tilde{V}(\bar{x}) \leq 0$ and thus 


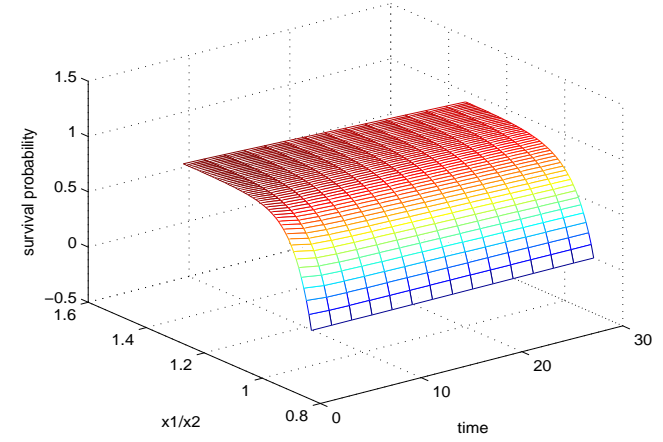

Fig. 8. Convergence of the survival probability for $r=0.08, \mu_{1}=0.06, \mu_{2}=0.05, \sigma_{1}=\sigma_{2}=0.25$, $\rho=0.3$ and $\lambda_{1}=0.9$.

$$
\begin{aligned}
E\left[e^{-r(\tau \wedge t)} \tilde{V}(X(\tau \wedge t))\right] \leq & \tilde{V}(\bar{x})-E \sum_{i=1}^{2} \int_{0}^{\tau \wedge t} e^{-r s} \frac{\partial \tilde{V}}{\partial x_{i}}(X(s)) \eta_{i} d C^{c}(s) \\
& -E \sum_{0 \leq s \leq \tau \wedge t} e^{-r s}\left[\tilde{V}(X(s))-\tilde{V}\left(X\left(s_{-}\right)\right)\right] .
\end{aligned}
$$

By concavity of $\tilde{V}(\bar{x})$, we can prove that $E\left[e^{-r(\tau \wedge t)} \tilde{V}(X(\tau \wedge t))\right] \rightarrow 0$ when $t \rightarrow+\infty$. Combined with $\nabla_{\eta} \tilde{V}(\bar{x}) \geq\left|\eta_{1}\right|+\left|\eta_{2}\right|$, it follows also that $\tilde{V}(X(s))-\tilde{V}\left(X\left(s_{-}\right)\right) \geq$ $\sum_{i=1}^{2} \frac{\partial \tilde{V}}{\partial x_{i}}\left(X_{i}(s)-X_{i}\left(s_{-}\right)\right) \geq\left(\left|\eta_{1}\right|+\left|\eta_{2}\right|\right) \Delta C(t)$. Since $C(t)=C^{c}(t)+\sum_{0 \leq s \leq t} \Delta C(s)$, we finally obtain the inequality

$$
\tilde{V}(\bar{x}) \geq E \int_{0}^{\tau} e^{-r s} \sum_{i=1}^{2}\left|\eta_{i}\right| d C(s)
$$

which is the first part of the result.

The local time $L_{t}^{e(\lambda)}(X)$ of the controlled process $X$ is a positive process that only increases when $X$ is located on the edge $e(\lambda)$ and maintains the firm value in the cone $O$. The second part follows from the same change of variable formula. As $(\mathcal{G}-r) \tilde{V}(\bar{x})=0$ when $\bar{x}$ is below the edge $e(\lambda)$, we directly obtain

$$
E\left[e^{-r(\tau \wedge t)} \tilde{V}(X(\tau \wedge t))\right]=\tilde{V}(\bar{x})-E \sum_{i=1}^{2} \int_{0}^{\tau \wedge t} e^{-r s} \frac{\partial \tilde{V}}{\partial x_{i}}(X(s)) \eta_{i} d L_{s}^{e(\lambda)}(X)
$$

Since $\int_{0}^{t} d L_{s}^{e(\lambda)}(X)=\int_{0}^{t} 1_{(X(s) \in e(\lambda))} d L_{s}^{e(\lambda)}(X)$ and $\nabla_{\eta} \tilde{V}(\bar{x})=\left|\eta_{1}\right|+\left|\eta_{2}\right|$ for $x \in e(\lambda)$, taking the limit $t \rightarrow+\infty$ provides

$$
\tilde{V}(\bar{x})=E_{\bar{x}}\left[\int_{0}^{\tau} e^{-r s} \sum_{i=1}^{2}\left|\eta_{i}\right| d L_{s}^{e(\lambda)}(X)\right]
$$




\section{A.2. Proof of Proposition 1}

We use similar arguments as in the first part of the proof of Theorem 2.8. in Burdzy et al. (2004). Consider the process $Y\left(X\left(t^{\prime}\right), t^{\prime}\right)$ defined by the formula

$$
Y\left(X\left(t^{\prime}\right), t^{\prime}\right)=e^{\alpha \int_{0}^{t^{\prime}} \sum_{i=1}^{2}\left|\eta_{i}\right| d L_{s}^{e(\lambda)}(X)} I\left(X\left(t^{\prime}\right), t-t^{\prime}\right),
$$

and assume that $I(\bar{x}, t)$ solves the partial differential equation. By standard use of Itô formula, we obtain

$$
\begin{aligned}
d Y\left(X\left(t^{\prime}\right), t^{\prime}\right)= & e^{\alpha \int_{0}^{t^{\prime}} \sum_{i=1}^{2}\left|\eta_{i}\right| d L_{s}^{e(\lambda)}(X)} \\
& \times\left(\alpha I\left(X\left(t^{\prime}\right), t-t^{\prime}\right) \sum_{i=1}^{2}\left|\eta_{i}\right| d L_{t^{\prime}}^{e(\lambda)}(X)+\left(\frac{d}{d t^{\prime}}+\mathcal{G}\right) I\left(X\left(t^{\prime}\right), t-t^{\prime}\right)\right. \\
& \left.+\sum_{i=1}^{2} \frac{\partial}{\partial x_{i}} I\left(X\left(t^{\prime}\right), t-t^{\prime}\right) \sigma_{i} X_{i}\left(t^{\prime}\right) d B_{i}\left(t^{\prime}\right)-\nabla_{\eta} I\left(X\left(t^{\prime}\right), t-t^{\prime}\right) d L_{t^{\prime}}^{e(\lambda)}(X)\right) \\
= & e^{\alpha \int_{0}^{t^{\prime}} \sum_{i=1}^{2}\left|\eta_{i}\right| d L_{s}^{e(\lambda)}(X)} \sum_{i=1}^{2} \frac{\partial}{\partial x_{i}} I\left(X\left(t^{\prime}\right), t-t^{\prime}\right) \sigma_{i} X_{i}\left(t^{\prime}\right) d B_{i}\left(t^{\prime}\right),
\end{aligned}
$$

as $\nabla_{\eta} I\left(\bar{x}, t-t^{\prime}\right)=\alpha \sum_{i=1}^{2}\left|\eta_{i}\right| I\left(\bar{x}, t-t^{\prime}\right)$ on $e(\lambda)$ and the local time $L_{t^{\prime}}^{e(\lambda)}(X)$ only increases on the edge $e(\lambda)$. We conclude that the process $\left\{Y\left(X\left(t^{\prime}\right), t^{\prime}\right), 0 \leq t^{\prime} \leq t\right\}$ is a local martingale. Moreover, adapting the arguments of Lemma 2.7. in Burdzy et al. (2004) to reflected geometric Brownian motion, we can prove that $Y$ is a bounded local martingale and thus a martingale :

$$
Y(\bar{x}, 0)=E_{\bar{x}}\left[e^{\alpha \int_{0}^{t} \sum_{i=1}^{2}\left|\eta_{i}\right| d L_{s}^{e(\lambda)}(X)} I(X(t), 0)\right],
$$

which completes the proof as $Y(\bar{x}, 0)=I(\bar{x}, t)$ and $I(X(t), 0)=1$. Finally the condition $I(\bar{x}, t)=1$ for all $\bar{x} \in e\left(\lambda_{1}\right)$ follows from the behavior of the process $X$ on the edge $e\left(\lambda_{1}\right)$.

\section{A.3. Proof of Proposition 2}

First, recall that there exists a line

$$
e(\lambda):=\left\{\bar{x}=\left(x_{1}, x_{2}\right) \in \mathbb{R}^{2}: x_{1}=\lambda x_{2}\right\}
$$

such that $\nabla_{\eta} \tilde{V}(\bar{x})>\sum_{i=1}^{2}\left|\eta_{i}\right|$ for $\bar{x}$ strictly below the line $e(\lambda)$, and $\nabla_{\eta} \tilde{V}(\bar{x})=\sum_{i=1}^{2}\left|\eta_{i}\right|$ for $\bar{x}$ on the line $e(\lambda)$, and thus $(\mathcal{G}-r) \tilde{V}(\bar{x})=0$ on $O$.

As a consequence, we can solve the ordinary differential equation $(\mathcal{G}-r) \tilde{V}(\bar{x})=0$ on $O$ subject to the boundary condition $\nabla_{\eta} \tilde{V}(\bar{x})=\sum_{i=1}^{2}\left|\eta_{i}\right|$ on the line $e(\lambda)$ for an arbitrary $\lambda$ and then optimize over $\lambda$, since $\tilde{V}(\bar{x})$ is the optimal gain function, see also Fleming and Soner, 1993.

We start to reduce the original HJB equation (6) using the scaling property

$$
\tilde{V}\left(x_{1}, x_{2}\right)=\left(x_{1}+x_{2}\right) \tilde{V}\left(\frac{x_{1}}{x_{1}+x_{2}}, \frac{x_{2}}{x_{1}+x_{2}}\right)=:\left(x_{1}+x_{2}\right) \tilde{W}(q)
$$


with $q=\frac{x_{1}}{x_{1}+x_{2}}$ and $\tilde{W}(q)=\tilde{V}(q, 1-q)$. A transformation of the derivatives in the HJB equation, finally leads to

$$
\begin{aligned}
\max \left\{\left(\eta_{1}(1-q)-\eta_{2} q\right) \frac{d \tilde{W}}{d q}(q)+\left(\eta_{1}+\eta_{2}\right) \tilde{W}(q)-\left(\left|\eta_{1}\right|+\left|\eta_{2}\right|\right),\right. & \\
\hat{\mathcal{G}} \tilde{W}(q)-r \tilde{W}(q)\} & =0
\end{aligned}
$$

together with the boundary condition $\tilde{W}\left(\lambda_{1} /\left(1+\lambda_{1}\right)\right)=0$, where $\hat{\mathcal{G}}$ is the operator

$$
\hat{\mathcal{G}}=d_{1}(q) \frac{d^{2}}{d q^{2}}+d_{2}(q) \frac{d}{d q}+d_{3}(q)
$$

with $d_{1}(q)=\frac{1}{2} \sigma^{2}(1-q)^{2} q^{2}, d_{2}(q)=\left(\mu_{1}-\mu_{2}\right) q(1-q)$, and $d_{3}(q)=\left(\mu_{1} q+\mu_{2}(1-q)\right)$.

We look for solutions in the form $\tilde{W}(q)=q^{\alpha}(1-q)^{\beta}$. The substitution of the suggested solution in the ordinary differential equation gives

$$
\begin{aligned}
{\left[\frac{1}{2} \alpha(\alpha-1) \sigma^{2}+\alpha\left(\mu_{1}-\mu_{2}\right)+\left(\mu_{2}-r\right)\right]+(\alpha+\beta-1) \times } \\
{\left[\frac{1}{2} \sigma^{2}(\alpha+\beta) q^{2}-\left(\alpha \sigma^{2}+\mu_{1}-\mu_{2}\right) q\right]=0 }
\end{aligned}
$$

and thus $\alpha$ solves the equation

$$
\frac{1}{2} \alpha(\alpha-1) \sigma^{2}+\alpha\left(\mu_{1}-\mu_{2}\right)+\left(\mu_{2}-r\right)=0
$$

and $\beta$ is determined by $\alpha+\beta-1=0$. The roots $\alpha_{1}$ and $\alpha_{2}$ are easily obtained

$$
\begin{aligned}
& \alpha_{1}=\frac{\frac{1}{2} \sigma^{2}-\left(\mu_{1}-\mu_{2}-\sqrt{\Delta}\right)}{\sigma^{2}} \\
& \alpha_{2}=\frac{\frac{1}{2} \sigma^{2}-\left(\mu_{1}-\mu_{2}+\sqrt{\Delta}\right)}{\sigma^{2}}
\end{aligned}
$$

with $\Delta=\frac{1}{4} \sigma^{4}+\left(\mu_{1}-\mu_{2}\right)^{2}-\sigma^{2}\left(\mu_{1}+\mu_{2}-2 r\right)$. Under the conditions $r>\mu_{2}$ and $\mu_{1}>\mu_{2}$, we have $\Delta>\left(\frac{1}{2} \sigma^{2}-\left(\mu_{1}-\mu_{2}\right)\right)^{2}>0$ and thus $\alpha_{1}<0$ and $\alpha_{2}>\alpha_{1}$. Moreover the additional condition $\mu_{1}>r$ implies $\Delta<\left(\frac{1}{2} \sigma^{2}+\left(\mu_{1}-\mu_{2}\right)\right)^{2}$ and $\alpha_{2}>1$. The general form of the solution is thus

$$
\tilde{V}\left(x_{1}, x_{2}\right)=C_{1} x_{1}^{\alpha_{1}} x_{2}^{1-\alpha_{1}}+C_{2} x_{1}^{\alpha_{2}} x_{2}^{1-\alpha_{2}} .
$$

The boundary condition $\tilde{V}(\bar{x})=0$ on $e\left(\lambda_{1}\right)$ leads to $C_{2}=-C_{1} \lambda_{1}^{\alpha_{1}-\alpha_{2}}$. We observe that $\nabla_{\eta} \tilde{V}(x)$ only depends on the ratio $\frac{x_{1}}{x_{2}}$ and we finally find the constant $C_{1}$ imposing the boundary condition $\nabla_{\eta} \tilde{V}(\bar{x})=\left|\eta_{1}\right|+\left|\eta_{2}\right|$ on $e(\lambda)$.

The optimal edge is derived by minimizing the denominator of $V(\bar{x})$ over $\lambda$. As the solutions to the indicial equation satisfy $\alpha_{1}<0$ and $\alpha_{2}>1$, it is easy to check that the denominator of $V(\bar{x})$ is the sum of two convex functions of $\lambda$ with a minimum because $\eta_{1}>0$ and $\eta_{2}<0$. Deriving with respect to $\lambda$ the denominator leads to the condition

$$
\begin{aligned}
& \eta_{1}\left(\alpha_{2}\left(\alpha_{2}-1\right) \lambda_{1}^{\alpha_{1}} \lambda^{\alpha_{2}-1}-\alpha_{1}\left(\alpha_{1}-1\right) \lambda_{1}^{\alpha_{2}} \lambda^{\alpha_{1}-1}\right) \\
& \quad-\eta_{2}\left(\alpha_{2}\left(\alpha_{2}-1\right) \lambda_{1}^{\alpha_{1}} \lambda^{\alpha_{2}}-\alpha_{1}\left(\alpha_{1}-1\right) \lambda_{1}^{\alpha_{2}} \lambda^{\alpha_{1}}\right)=0 .
\end{aligned}
$$

It is trivial to check that the value proposed for $\lambda_{*}$ satisfies the condition. 


\section{A.4. Proof of Proposition 3}

Default occurs whenever the firm value falls below the solvency limit $e\left(\lambda_{1}\right)$ with $\lambda_{1}<\lambda$. The time to default satisfies

$$
\tau=\inf \left\{t: X(t) \in e\left(\lambda_{1}\right)\right\}=\inf \left\{t: \frac{X_{1}(t)}{X_{2}(t)}=\lambda_{1}\right\} .
$$

The process $Y_{1}(t \wedge \tau)=X_{1}(t \wedge \tau) / X_{2}(t \wedge \tau)$ is a linear diffusion as well, with infinitesimal generator given by

$$
\mathcal{K}=\frac{1}{2} \hat{\sigma}_{1,1}^{2} y^{2} \frac{\partial^{2}}{\partial y^{2}}+\hat{\mu}_{1} y \frac{\partial}{\partial y},
$$

acting on the domain $\mathcal{D}=\left\{u: u, \mathcal{K} u \in C_{b}\left(\left(\lambda_{1}, \lambda\right]\right), u\left(\lambda_{1}+\right)=0, u^{\prime}(\lambda)=0\right\}$. As the state-space $\left(\lambda_{1}, \lambda\right]$ is bounded, the spectral representation for the transition density of $Y_{1}$ reduces to a series expansion, see Itô and McKean (1974) or Linetsky (2004). The survival probability $P_{z}(t)$ satisfies the following partial differential equation

$$
\frac{\partial}{\partial t} P_{z}(t)=\mathcal{K} P_{z}(t), \quad z \in\left(\lambda_{1}, \lambda\right]
$$

subject to the boundary conditions

$$
P_{z}(0)=1, \quad P_{\lambda_{1}}(t)=0, \quad \frac{\partial}{\partial z} P_{\lambda_{1}}(t)=0 .
$$

The self-adjoint operator $\mathcal{K}$ acts on the set of $w$-square integrable functions and the ordinary differential equations $\mathcal{K} \phi(z)=-E \phi(z)$ together with the conditions $\phi\left(\lambda_{1}\right)=0$ and $\frac{d}{d z} \phi(\lambda)=0$ is a Sturm-Liouville problem generating a discrete sequence of eigenvalues $\left\{E_{n}, n \in \mathcal{N}\right\}$ and eigenfunctions $\phi_{n}$ orthonormal with respect to the scalar product

$$
\langle f, g\rangle=\int_{\lambda_{1}}^{\lambda} f(z) g(z) w(z) d z .
$$

We observe that the functions $z^{\theta} \cos (b \ln x)$ and $z^{\theta} \sin (b \ln x)$ span the oscillating solutions of $\mathcal{K} u=-E u$ and we construct the eigenfunction $\phi_{n}$ as a linear combination:

$$
\phi_{n}(z)=\beta_{1} z^{\theta} \cos (b \ln z)+\beta_{2} z^{\theta} \sin (b \ln z)
$$

to some normalizing constant, the condition $\phi_{n}\left(\lambda_{1}\right)=0$ provides the constant and the condition $\frac{d}{d z} \phi_{n}(\lambda)=0$ the eigenvalues. The function $P_{z}(t)$ decomposes into the series expansion

$$
P_{z}(t)=\sum_{n=0}^{+\infty} c_{n} e^{-E_{n} t} \phi_{n}(z),
$$

the coefficients $c_{n}$ are chosen to satisfy the initial condition

$$
P_{z}(0)=1=\sum_{n=0}^{+\infty} c_{n} \phi_{n}(z) .
$$

\section{A.5. Proof of Proposition 4}

In the coordinates (15), the partial differential equation (14) can be simplified by means of the scaling property of $V$, or 


$$
V\left(\left(x_{1}, x_{2}\right), t\right)=x_{2} V\left(\left(\frac{x_{1}}{x_{2}}, 1\right), t\right)=: y_{2} V\left(\left(y_{1}, 1\right), t\right) .
$$

By means of a transformation of the derivatives, the two-dimensional partial differential equation $\frac{\partial}{\partial t} V(\bar{x}, t)=(\mathcal{G}-r) V(\bar{x}, t)$ reduces to a scalar equation for the quantity $W(z, t)$ (with $z=x_{1} / x_{2}$ )

$$
\frac{\partial}{\partial t} W(z, t)=\left[\frac{\sigma^{2}}{2} z^{2} \frac{\partial^{2}}{\partial z^{2}}+\left(\mu_{1}-\mu_{2}\right) z \frac{\partial}{\partial z}+\left(\mu_{2}-r\right)\right] W(z, t), \quad z=\frac{x_{1}}{x_{2}}
$$

subject to the boundary conditions

$$
W(z, 0)=0, \quad W\left(\lambda_{1}, t\right)=0, \quad \frac{\partial}{\partial z} W(\lambda, t)=1 .
$$

See also Gerber and Shiu (2003) who originally obtain this equation using martingale arguments, the sampling theorem more precisely. We rely on the spectral decomposition of the self-adjoint linear operator

$$
\hat{\mathcal{K}}=\frac{\sigma^{2}}{2} z^{2} \frac{\partial^{2}}{\partial z^{2}}+\left(\mu_{1}-\mu_{2}\right) z \frac{\partial}{\partial z}+\left(\mu_{2}-r\right)
$$

on the bounded domain $\left(\lambda_{1}, \lambda\right]$ to derive a series expansion for $W(z, t)$. Define the surplus $J(z, t)=\breve{V}(z)-W(z, t)$. It is easy to check that $J(z, t)$ is the solution to the partial differential equation

subject to the boundary conditions

$$
\frac{\partial}{\partial t} J(z, t)=\hat{\mathcal{K}} J(z, t), \quad z \in\left(\lambda_{1}, \lambda\right]
$$

$$
J(z, 0)=\breve{V}(z), \quad J\left(\lambda_{1}, t\right)=0, \quad \frac{\partial}{\partial z} J(\lambda, t)=0 .
$$

The self-adjoint operator $\hat{\mathcal{K}}$ acts on the set of $w$-square integrable functions and the ordinary differential equations $\hat{\mathcal{K}} \phi(z)=-E \phi(z)$ together with the conditions $\phi\left(\lambda_{1}\right)=0$ and $\frac{d}{d z} \phi(\lambda)=0$ is a Sturm-Liouville problem generating a discrete sequence of eigenvalues $\left\{E_{n}, n \in \mathcal{N}\right\}$ and eigenfunctions $\phi_{n}$ orthonormal with respect to the scalar product

$$
\langle f, g\rangle=\int_{\lambda_{1}}^{\lambda} f(z) g(z) w(z) d z .
$$

We observe that the functions $z^{\theta} \cos (b \ln x)$ and $z^{\theta} \sin (b \ln x)$ span the oscillating solutions of $\hat{\mathcal{K}} u=-E u$ and we construct the eigenfunction $\phi_{n}$ as a linear combination:

$$
\phi_{n}(z)=\beta_{1} z^{\theta} \cos (b \ln z)+\beta_{2} z^{\theta} \sin (b \ln z)
$$

to some normalizing constant, the condition $\phi_{n}\left(\lambda_{1}\right)=0$ provides the constant and the condition $\frac{d}{d z} \phi_{n}(\lambda)=0$ the eigenvalues. The function $J(z, t)$ decomposes into the series expansion

$$
J(z, t)=\sum_{n=0}^{+\infty} c_{n} e^{-E_{n} t} \phi_{n}(z),
$$

the coefficients $c_{n}$ are chosen to satisfy the initial condition

$$
J(z, 0)=\breve{V}(z)=\sum_{n=0}^{+\infty} c_{n} \phi_{n}(z)
$$




\section{References}

[1] Abramowitz M., \& Stegun I.A. (1970). Handbook of mathematical functions, Dover books on mathematics, New York, $1046 \mathrm{p}$.

[2] Asmussen S., \& Taksar M. (1997). "Controlled diffusion models for optimal dividend pay-off", Insurance: Mathematics and Economics, vol.20, p.1-15.

[3] Black F. \& Cox J. (1976). "Valuing corporate securities : some effects of bond indenture provisions", Journal of Finance, vol.31(May), p.351-67.

[4] Burdzy K. , Zhin-Qing Chen \& Sylvester J. (2004). "The heat equation and reflected Brownian motion in time-dependent domains", The Annals of Probability, Vol.32, p.775-804.

[5] Davis M.H.A. \& Norman A.R (1990). "Portfolio selection with transactions costs", Mathematics of Operations Research, vol.15(4), p.676-713.

[6] Fleming W.H. \& Soner H.M. (1993). Controlled Markov processes and viscosity solutions, SpringerVerlag, New York, 428 p.

[7] Gerber H., \& Shiu S.W. (2003). "Geometric Brownian motion models for assets and liabilities: from pension funding to optimal dividends", North American Actuarial Journal, vol.7(3), p.37-51.

[8] Gerber H., \& Shiu S.W. (2004). "Optimal dividends: analysis with Brownian motion", North American Actuarial Journal, vol.8(1), p.1-20.

[9] Grandits P., Hubalek F., Schachermayer W., \& Zigo M. (2007). "Optimal expected exponential utility of dividend payments in a Brownian risk model", Scandinavian Actuarial Journal, to appear.

[10] Harrison J.M. \& Taksar M. (1983). "Instantaneous Control of Brownian Motion", Mathematics of Operations Research, vol.8, p.439-453.

[11] Hoevenaers R.P.M.M., Molenaar R.D.J., Schotman P.C. \& Steenkamp T.B.M. (2008). "Strategic asset allocation with liabilities: Beyond stocks and bonds", Journal of Economic Dynamics and Control, vol.32, p.2939-2970.

[12] Hojgaard B. \& Taskar M. (2002). "Optimal dynamic portfolio selection for a corporation with controllable risk and dividend policy", Working paper.

[13] Hojgaard B. (2002). "Optimal dynamic premium control in non-life insurance: maximizing dividends pay-outs", Scandinavian Actuarial Journal, p.225-245.

[14] Hojgaard B. \& Taksar (1999). "Controlling risk exposure and dividends payout schemes: insurance company example", Mathematical Finance, vol.9, p.153-182.

[15] Hubalek F. \& Schachermayer W. (2004). "Optimizing expected utility of dividend payments for a Brownian risk process and a peculiar nonlinear ODE", Insurance: Mathematics and Economics, vol.34(2), p.193-225.

[16] Itô K., \& McKean H.P. (1965). Diffusion processes and their sample paths, Academic Press, New York, $321 \mathrm{p}$.

[17] Leland H.E. (1994). "Corporate debt value, bond covenants and optimal capital structure", Journal of Finance, vol.49(4), p.1213-1252.

[18] Lewis A. (1998). "Applications of eigenfunction expansions in continuous-time finance", Mathematical Finance, vol.8, p.349-383.

[19] Linetsky V. (2004): "The spectral decomposition of the option value", International Journal of Theoretical and Applied Finance, vol.7(3), p.337-384.

[20] Linetsky V. (2004): "Spectral expansions for Asian (Average Price) Options", Operations Research, vol.52, p.856-867.

[21] Linetsky V. (2004): "Lookback options and diffusion hitting times: A spectral expansion approach", Finance and Stochastics, vol.8, p.373-398.

[22] Lions P.L. \& Sznitman A.S. (1984). "Stochastic differential equations with reflecting boundary conditions", Communications on Pure and Applied Mathematics, vol.37(4), p.511-537.

[23] Modigliany, F. \& Miller M. (1958). "The cost of capital, corporation finance and the theory of investments", American Economic Review, vol.48, p.267-97.

[24] Revuz D., \& Yor M. (1998). Continuous martingales and Brownian motion, Springer-Verlag, Berlin, 533 p.

[25] Rudolf M. \& Ziemba W.T. (2004). "Intertemporal surplus management", Journal of Economic Dynamics and Control, vol.28, p.975-990.

[26] Taksar M.I. (2000). "Optimal risk and dividend distribution models for an insurance company. Mathematical methods of operations research, vol.51(1), p.1-42. 